\title{
Continuous fabrication of near-infrared light responsive bilayer hydrogel fibers based on microfluidic spinning
}

https://doi.org/10.1515/epoly-2019-0022

Received October 18, 2018; accepted November 12, 2018.

\begin{abstract}
Hydrogel microfibers with inhomogenous structure can achieve some complex motions such as bending, folding and twisting. So it can be applied to soft actuators, soft robots and micropumps. In this paper, continuous bilayer hydrogel fibers in which one layer is calcium alginate hydrogel and the other is linear poly(N-isopropylacrylamide) (PNIPAM)/calcium alginate/graphene oxide (GO) semi-interpenetrating hydrogel were prepared based on microfluidic spinning method. The results show that the bilayer hydrogel fibers have particular porous internal structures of semi-IPN hydrogels and the pore size becomes smaller with the increase of GO content. Besides, the bilayer hydrogel fibers can bend response to the temperature and near-infrared (NIR) light. The diameter of the hydrogel fibers can be tuned by changing the flow rate of spinning fluid and the take-up velocity of winding device.
\end{abstract}

Keywords: bilayer hydrogel fiber; microfluidic spinning; NIR light responsive; calcium alginate; poly(N-isopropylacrylamide)

\section{Introduction}

Stimuli-responsive hydrogels as a kind of smart material can change their volumes in response to various stimuli such as temperature (1), $\mathrm{pH}(2)$, electric field $(3,4)$ and

\footnotetext{
* Corresponding authors: Jinghua Gong and Jinghong Ma, State Key Laboratory for Modification of Chemical Fibers and Polymer Materials, College of Materials Science and Engineering, Donghua University, Shanghai, 201620, China, email: gjh@dhu.edu.cn; mjh68@dhu.edu.cn

Meiling Zhou, State Key Laboratory for Modification of Chemical Fibers and Polymer Materials, College of Materials Science and Engineering, Donghua University, Shanghai, 201620, China
}

glucose concentrations (5). Stimuli-responsive hydrogels with homogeneous structure usually show reversible isotropic volume expansion or contraction. Recently, considerable efforts have been focused on the fabrication of inhomogeneous hydrogels which can achieve some complex motions such as bending, twisting and folding by controlling the external stimulus. They provide more possibilities to fabricate artificial muscle, soft actuators and micropumps (6).

Until now, various stimuli-responsive hydrogels with inhomogeneous structure have been prepared by many methods including stacking stimuli-responsive hydrogels to form multilayer hydrogel sheets (7), forming hydrogel microfibers or microspheres with complex structure by microfluidics device $(8,9)$, forming bilayer fibers or films by electrospinning (10-12) and in-situ polymerization under special external conditions (13). Among these, microfluidic spinning and electrospinning are facile ways to obtain bilayer fibers. For example, a bilayer fiber composed of poly( $\mathrm{N}$-isopropylacrylamide) (PNIPAM) and poly(methyl methacrylate-co-butyl methacrylate) was prepared by electrospinning (10). After photo-crosslinking, the prepared bilayer fiber can achieve reversible buckling and stretching in response to temperature in water. Recently, a one-step and continuous spinning method based on fast ion-crosslinking was reported for preparing bilayer hydrogel. Yao et al. fabricated a bilayer sodium alginate (SA) hydrogel fiber using a multi-channel capillary microfluidics and applied the complex microfiber for cell co-culture and 3D growth (9). Shunsuke et al. prepared a bilayer $\mathrm{SA} /$ poly(N-isopropylacrylamide-co-acrylic acid) hydrogel fiber by using a microfluidic spinning device containing a Y-shaped connector (8). The bilayer hydrogel fiber can bend and unbend in response to temperature and $\mathrm{pH}$, and has potential application in the soft actuators and robots.

Recently, NIR light responsive hydrogels have attracted wide interest because NIR light is easy to be controlled. NIR light responsive hydrogels were usually prepared by 
combining thermo-sensitive polymer such as PNIPAM with light-absorbing nanoparticles such as carbon nanotubes (14), graphene (15), graphene oxide (16-18), gold nanorods $(19,20)$ and ferroferric oxide nanoparticales $(21,22)$. Graphene oxide has the advantages of high NIR light absorbance and conversion efficiency. Consequently, GO has been utilized not only in robust polymer hydrogels but also in photoresponse functional hydrogels. Zhang et al. designed a new IR driving hydrogel by stacking GO/PNIPAM hydrogel sheet and PNIPAM hydrogel sheet to form a bilayer hydrogel (7). The bilayer hydrogel showed a rapid, reversible and tunable anisotropic bending in response to the NIR irradiation.

However, there is no report on the preparation of NIR light responsive hydrogels fiber based on microfluidic spinning. Here, we present a one-step, continuous process for the hydrogel fibers with bilayer structure by using a microfluidic spinning device containing a Y-shaped channel. Water-soluble linear PNIPAM were first synthesized by free radical polymerization reaction. Then bilayer hydrogel fibers with one layer of calcium alginate hydrogel and the other layer of PNIPAM/calcium alginate/GO semi-IPN hydrogel were prepared. The pore structure, mechanical property, swelling ratio and temperature/NIR light responsive behaviors were studied.

\section{Materials and methods}

\subsection{Materials}

$N$-Isopropylacrylamide (NIPAM, TCI Co., Ltd.) was purified by recrystallization from toluene/cyclohexane
(6/4, v/v) to remove inhibitor before use. Potassium persulfate (KPS, Shanghai Chemical Co., Ltd.) was purified by recrystallization in deionized water. $N, N, N^{\prime}, N^{\prime}-$ tetramethylethylenediamine (TEMED) and sodium alginate (SA) were purchased from Aladdin Reagent Co., Ltd. Calcium chloride $\left(\mathrm{CaCl}_{2}\right)$ was obtained from Shanghai Chemical Co., Ltd. Graphene oxide (GO) was obtained from Nanjing XFNANO Materials Technology Co., Ltd. Poly(dimethylsiloxane) (PDMS) prepolymer and curing agent were obtained from Dow Corning.

\subsection{Microfluidic device}

In this paper, the microfluidic spinning device was designed and assembled based on the report of Shunsuke et al. (8). As is shown in Figure 1, the microfluidic spinning device consists of Y-shaped channel, conical tube, collection channel and winding device. The Y-shaped channel ensures that two fluids can form a stable parallel laminar flow after their meeting. The conical tube and the outer fluidic channel make it easier to control the diameter of hydrogel fiber. The function of winding device is to collect the continuous hydrogel fiber. The sizes of each part are shown in Figure 1.

\subsection{Preparation and characterization of PNIPAM}

NIPAM (5 g) monomer and deionized water (50 mL) were mixed and stirred in $100 \mathrm{~mL}$ flask till complete dissolution. The flask was placed into ice-water bath and the NIPAM solution was purged with nitrogen for $30 \mathrm{~min}$ to remove oxygen. Then KPS solution $(0.2 \mathrm{~g} / \mathrm{mL}$, $0.5 \mathrm{~mL})$ and TEMED $(50 \mu \mathrm{L})$ were added to initiate the
A

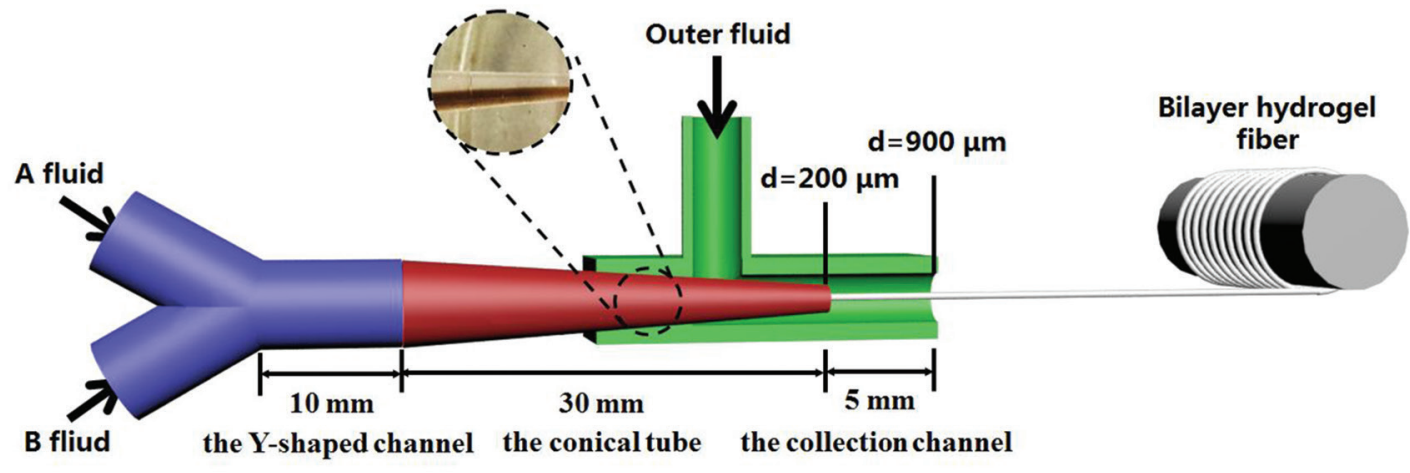

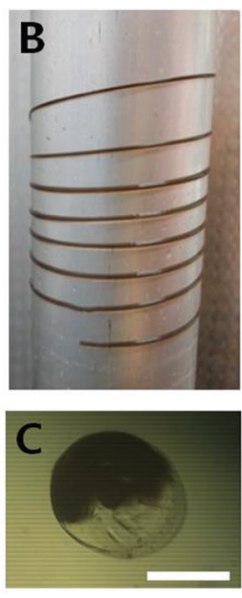

Figure 1: Schematic diagram of microfluidic spinning device. scale bar of SEM images is $500 \mu \mathrm{m}$. 
polymerization reaction. After $2 \mathrm{~h}$, the reaction solution was taken out and heated to $80^{\circ} \mathrm{C}$. Then the precipitate was collected and dried at $50^{\circ} \mathrm{C}$.

Dry polymers were characterized byFTIR spectroscopy (Nicolet 6700) and its molecular mass was analyzed by GPC (PL-GPC 50 Agilent, LS) in dimethylacetamide at $20^{\circ} \mathrm{C}$.

\subsection{Preparation of bilayer hydrogel fiber}

Three kinds of solution were used in the preparation of bilayer hydrogel fibers. A fluid was an aqueous solution composed of linear PNIPAM, SA and GO, in which the concentrations of PNIPAM and SA were $15 \mathrm{mg}$ $\mathrm{mL}^{-1}$ and $10 \mathrm{mg} \mathrm{mL}^{-1}$, respectively. GO was ultrasonically dispersed in deionized water, and then $\mathrm{NaOH}$ was added to adjust the $\mathrm{pH}$ value of the solution to 7.0. The concentrations of $\mathrm{GO}$ in A fluid were designed as $n\left(n=0,1,2\right.$ and $\left.4 \mathrm{mg} \mathrm{mL}^{-1}\right)$. B fluid is SA aqueous solution with concentration of $20 \mathrm{mg} \mathrm{mL}^{-1}$. The outer fluid is $\mathrm{CaCl}_{2}$ aqueous solution with concentration of $1 \mathrm{M}$. A fluid, B fluid and $\mathrm{CaCl}_{2}$ solution were introduced into microfluidic spinning device by syringe pumps (PHD ULTRA, Harvard Apparatus) as shown in Figure 1. The hydrogel fiber out of the channel was collected by the winding device. The hydrogel fiber has bilayer structure, one is calcium alginate hydrogel layer, and the other is PNIPAM/calcium alginate/GO semi-IPN hydrogel layer. Therefore, the bilayer hydrogel fibers are named as $C / C P G_{n}$ hydrogel fibers, in which $n$ represents the concentrations of GO in A fluid.

\subsection{Morphology observation}

The swollen hydrogel fibers were quickly frozen in liquid nitrogen and then freeze-dried at $-48^{\circ} \mathrm{C}$ for $24 \mathrm{~h}$. Then the hydrogel fibers was broken in liquid nitrogen. The microstructure of the cross section was observed with scanning electron microscope (SEM, SU8010, Hitachi). The optical microscope photograph of the surface and cross section of hydrogel fibers was observed with optical microscope (XSP-17C, Shanghai Gauangmi Instrument Co., Ltd.).

\subsection{Mechanical property}

The mechanical property of hydrogel fibers was evaluated by tensile stress measurement using an Instron 5900 testing machine at room temperature.
The diameter of hydrogel fibers is $500 \mu \mathrm{m}$. The distance between the two clamps is $20 \mathrm{~mm}$ and the tensile speed is $20 \mathrm{~mm} \mathrm{~min}^{-1}$.

\subsection{Equilibrium swelling ratio}

The hydrogel fibers with the same diameter and length were immersed into deionized water to approach the swelling equilibrium at room temperature. After wiping away the excess water on the surface with filter paper, the mass of the swollen hydrogel was measured at a time interval. The swelling ratio $(S R)$ of the hydrogel was calculated as the follows:

$$
S R=\left(W_{s}-W_{d}\right) / W_{d}
$$

where $W_{s}$ and $W_{d}$ represent the weights of hydrogel fibers in the swollen state and dry state, respectively.

\subsection{Temperature/NIR light responsive behavior}

The hydrogel fibers were immersed into deionized water to reach the swelling equilibrium at room temperature and cut into $1 \mathrm{~cm}$ in length for the actuation test. The temperature responsive behavior was observed in $50^{\circ} \mathrm{C}$ of deionized water. The NIR light responsive behavior was observed in the atmosphere. The NIR light laser $(808 \mathrm{~nm})$ was used as the light source located at a distance of $20 \mathrm{~cm}$ from the fibers. The power density delivered to the sample was $3 \mathrm{~W} \mathrm{~cm}-2$. The temperature/NIR light responsive behaviors of the hydrogel fibers were recorded by a camera.

\subsection{The characterization of single layer hydrogel fiber}

For further study of the effect of $\mathrm{GO}$ content on the property of PNIPAM/calcium alginate/GO hydrogel, the single layer hydrogel fibers (named as $\mathrm{CPG}_{\mathrm{n}}$ ) were prepared and their chemical composition are the same with the layer $\mathrm{A}$ of the $\mathrm{C} / \mathrm{CPG}_{\mathrm{n}}$ hydrogel fibers, respectively. Its volume-phase transition temperature (VPTT) of the hydrogel fibers was tested using a differential scanning calorimeter (DSC, 204F1, Nicolet) from $20^{\circ} \mathrm{C}$ to $45^{\circ} \mathrm{C}$ at a constant heating rate of $1^{\circ} \mathrm{C} \mathrm{min}^{-1}$ under a nitrogen atmosphere with a flow rate of $50 \mathrm{~mL} \mathrm{~min}^{-1}$. The temperature of $\mathrm{CPG}_{\mathrm{n}}$ single layer hydrogel fibers under the irradiation of NIR light (808 nm, $3 \mathrm{~W} \mathrm{~cm}^{-2}$ ) were measured using a infrared thermal imager. 


\section{Results and discussion}

\subsection{Preparation and characterization of PNIPAM}

Linear PNIPAM polymer was synthesized by free radical polymerization initiated by KPS in water (23-25). In the polymerization process, the reaction solution became very viscous after $1 \mathrm{~h}$. In order to confirm the phase transition characteristic of the synthetic product, the reaction solution was heated to $50^{\circ} \mathrm{C}(>\mathrm{LCST})$ and cooled to $0^{\circ} \mathrm{C}(<\mathrm{LCST})$ in succession. It was found that the solution turned to turbid at $50^{\circ} \mathrm{C}$ and became clear and transparent at $0^{\circ} \mathrm{C}$. In addition, the FTIR spectrum (Figure 2a) exhibits characteristic peaks at 1645 and $1540 \mathrm{~cm}^{-1}$ corresponding to amide I and amide II, respectively. The characteristic peaks at 2877,2931 and $2972 \mathrm{~cm}^{-1}$ corresponding to stretching vibration of $\mathrm{C}-\mathrm{H}$ confirm that the synthetic polymer is linear PNIPAM (26). GPC result shows that the weightaverage molar mass of PNIPAM is $3.0 \times 10^{5} \mathrm{~g} \mathrm{~mol}^{-1}$ and its polydispersity index $(P D I)$ is 2.0 , which is consistent with the report on PNIPAM synthesized by one-step radical polymerization $(1,27)$.

\subsection{Preparation and characterization of hydrogel fiber}

The bilayer hydrogel fibers were prepared by using a microfluidic spinning device with Y-shaped channel. As is shown in Figure 1, the spinning solutions (A fluid

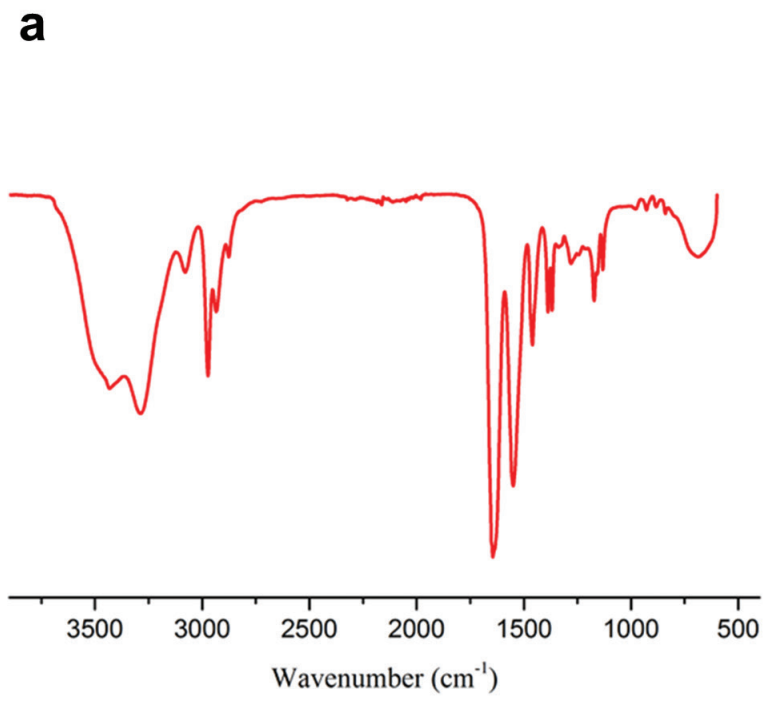

and $\mathrm{B}$ fluid) and the $\mathrm{CaCl}_{2}$ solution were introduced into the inner and outer fluidic channels at a certain flow by syringe pumps, respectively. A fluid and B fluid met in the Y-shaped channel and formed a laminar flow in the conical tube because of their low Reynolds number. When they flowed into the collection channel and formed a stable coaxial flow with $\mathrm{CaCl}_{2}$ solution, $\mathrm{Ca}^{2+}$ ions diffused into both inner A and B fluids quickly. Because the guluronate units in the different free SA chains can form coordinate structure called as the "egg-box" junction, the A and B fluids were solidified by an ionic crosslinking stably and rapidly in the stable coaxial flow of inner fluids and $\mathrm{CaCl}_{2}$ solution (28). Based on this feature, the spinning fluids can form straight fiber immediately because of the fast ionic crosslinking of alginate and $\mathrm{Ca}^{2+}$.

Because of the different composition for A and B fluid, two parallel layers were formed in the hydrogel fiber. One is calcium alginate hydrogel layer, and the other is PNIPAM/ calcium alginate/GO semi-IPN hydrogel layer. Only the PNIPAM/calcium alginate/GO semi-IPN hydrogel layer has temperature and NIR light responsive property. The anisotropic swelling/shrinking in respective to the external stimulus makes the bilayer hydrogel fibers achieve more complex motions such as bending, twisting and folding.

Optical microscope photograph of the bilayer hydrogel fiber is shown in Figure 3a. It can be found that two different layers are aligned along the axial direction of the hydrogel fiber. Layer A is PNIPAM/calcium alginate/GO semi-IPN hydrogel layer, and layer B is calcium alginate hydrogel layer. The layer A is black and non-transparent because of the existence of GO. Corresponding to the microscope photograph, SEM images of the cross section for layers A and B of hydrogel fiber (Figures $3 b-i$ ) show the

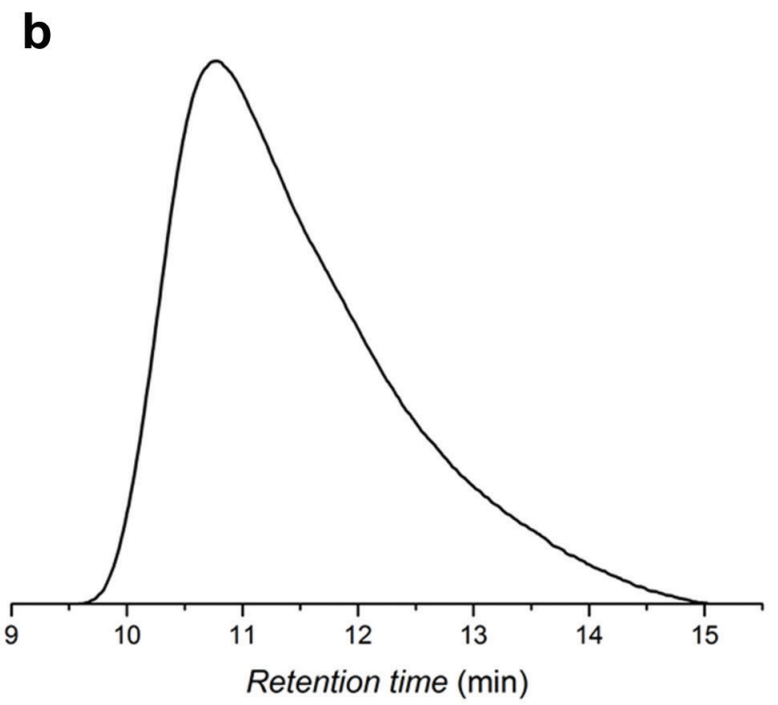

Figure 2: FTIR spectrum (a) and GPC trace (b) of linear PNIPAM. 
difference in the morphology and pore size between layer A and layer B. As shown in Figures 3f-i, the morphology and size of the pores in layer B hydrogel are uniform because all composition of the spinning solution for layer $\mathrm{B}$ are the same, which leads to the identical hydrogel network formed during the microfluidic spinning.

The SEM images of the cross section for layer A hydrogel with different GO contents are shown in Figures 3b-e. Compared with layer B, the pore density is lower and the pore size is nonuniform. As mention above, SA chains were crosslinked by $\mathrm{Ca}^{2+}$ ions to form hydrogel network with linear PNIPAM interpenetrated in it. The concentration of $\mathrm{SA}$ in layer A is smaller than that in layer B, resulting to the lower crosslinking density, and linear PNIPAM has influence on the pore uniformity. In addition, with increasing the GO content, pore size becomes smaller, implying that a denser network structure has been formed in the layer A of hydrogel. The dense network can be attributed to the hydrogen bonding interaction between the $\mathrm{N}-\mathrm{H}$ bond of PNIPAM and the O-H bond of GO nanosheet (29).

\subsection{Mechanical property}

The research of Shen et al. showed the GO sheets in the Polyacrylamide/GO hydrogel can strengthen the tensile properties of hydrogel because it can form the physical crosslinking structure in the polymer matrix (30). Figure 4 is the stress-strain curve for the bilayer hydrogel fiber with
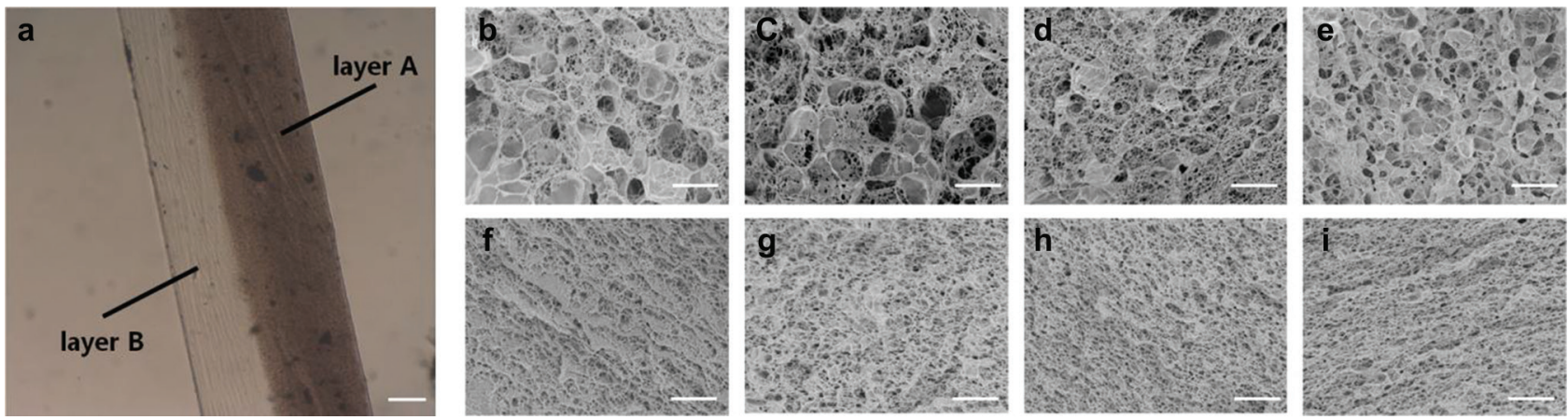

Figure 3: (a) Optical microscope photograph of the surface and (b-i) SEM images of cross section of hydrogel fibers. Scale bar of microscope photograph is $100 \mu \mathrm{m}$, scale bar of SEM images is $2 \mu \mathrm{m}$. B-E and F-I are the layer $A$ and layer $B$ for $C / C P G_{0}, C / C P G_{1}, C / C P G_{2}$ and $\mathrm{C} / \mathrm{CPG}_{4}$ hydrogel fiber, respectively.

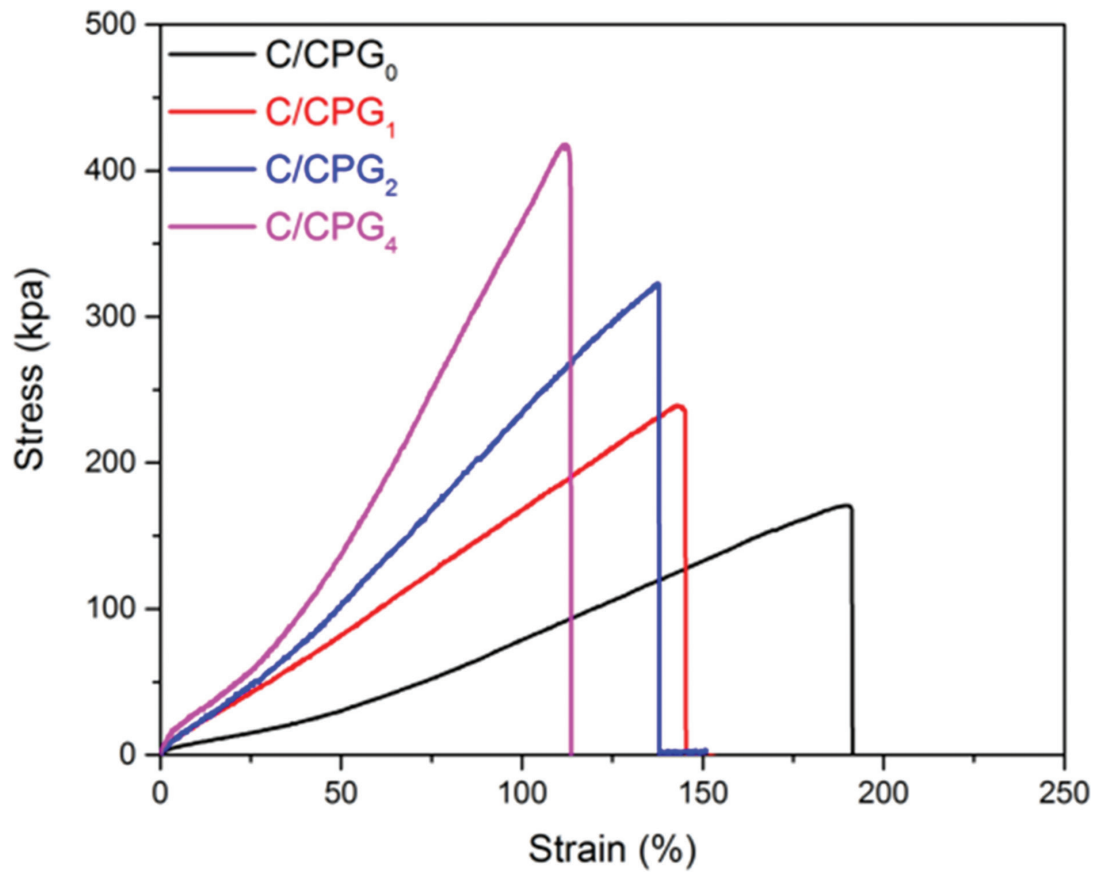

Figure 4: The tensile stress-strain curve of the bilayer hydrogel fibers. 
$425 \mu \mathrm{m}$ in diameter. It can be seen that with the increase of GO contents, the strength and modulus of the hydrogel fibers improve significantly, while the elongation at break decreases. The $\mathrm{C} / \mathrm{CPG}_{4}$ hydrogel fiber has good mechanical strength and its breaking strength reaches $400 \mathrm{kPa}$, which is due to the increase of the crosslink density of the hydrogel by embedding GO into the hydrogel matrix.

\subsection{Swelling behavior}

Figure 5 is the swelling curve of the bilayer hydrogel fibers in deionized water at $20^{\circ} \mathrm{C}$. The swelling ratios of the hydrogel fibers increase rapidly at the early stage, and then reach their equilibrium states. In the progress of hydrogel swelling, the GO sheets in the hydrogel have complicated influence on the equilibrium swelling ratios. On the one hand, the GO sheets increase the crosslink density of polymer matrix and impede the swelling of hydrogel fiber. On the other hand, they improve the hydrophilicity of hydrogel fiber because the GO sheets contain some hydrophilic groups $(31,32)$. It can be found from the comparison of $\mathrm{C} / \mathrm{CPG}_{1}, \mathrm{C} / \mathrm{CPG}_{2}$ and $\mathrm{C} / \mathrm{CPG}_{4}$ hydrogel fibers that the equilibrium swelling ratios reduce with the increase of GO content. The phenomenon is attributed to the fact that the polymer network structure of hydrogel fiber is more compact with the increase of the
GO content. However, all of them are larger than that of $\mathrm{C} / \mathrm{CPG}_{0}$ sample, because $\mathrm{GO}$ sheets in the hydrogel matrix contain some hydrophilic groups, resulting that more water can be held in the polymer network.

\subsection{Temperature responsive bending behavior}

Figure $6 \mathrm{a}$ is the schematic of the temperature or NIR light responsive bending of the bilayer hydrogel fiber. The bilayer hydrogel fiber with length in $1.0 \mathrm{~cm}$ was fixed at one end and put in deionized water at $50^{\circ} \mathrm{C}$ to observe temperature responsive bending behavior. As shown in Figure $6 \mathrm{~b}$, the $\mathrm{C} / \mathrm{CPG}$ fiber bent toward layer $\mathrm{A}$ and the bending angle increased gradually with prolonging the response time. Because thermosensitive polymer PNIPAM is only distributed in layer A of the hydrogel fiber, layer A will shrinkage at the temperature above VPTT, while layer B does not response to temperature. As a result, the hydrogel fiber will bend toward layer A (Figure 6b).

The VPTT of hydrogel fibers was measured by DSC (Figure 7). The result shows that the incorporation of GO has limited influence on the VPTT of the hydrogel fibers because of no chemical bond formed between PNIPAM network and GO nanosheet.



Figure 5: The swelling curve of the hydrogel fiber in deionized water at $20^{\circ} \mathrm{C}$. 

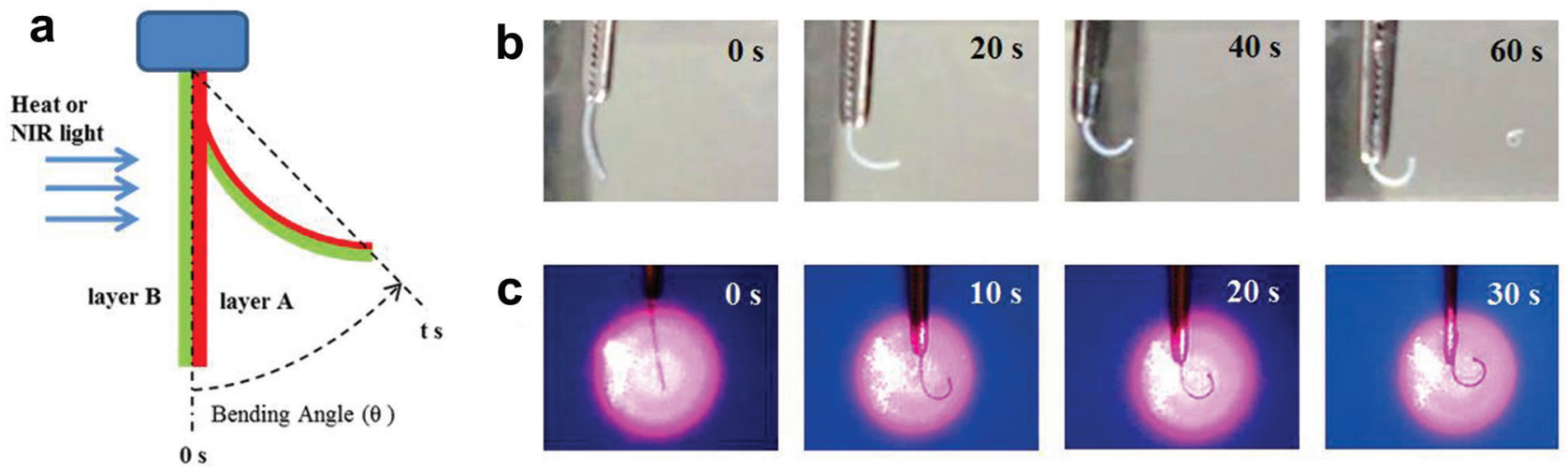

Figure 6: (a) Schematic of the temperature/NIR light responsive bending of the bilayer hydrogel fiber. (b) The photographs of temperature responsive bending of the $\mathrm{C} / \mathrm{CPG}_{0}$ fiber in deionized water at $50^{\circ} \mathrm{C}$. (c) The photographs of NIR light responsive bending of the $\mathrm{C} / \mathrm{CPG} 2$ fiber after irradiated by NIR light.

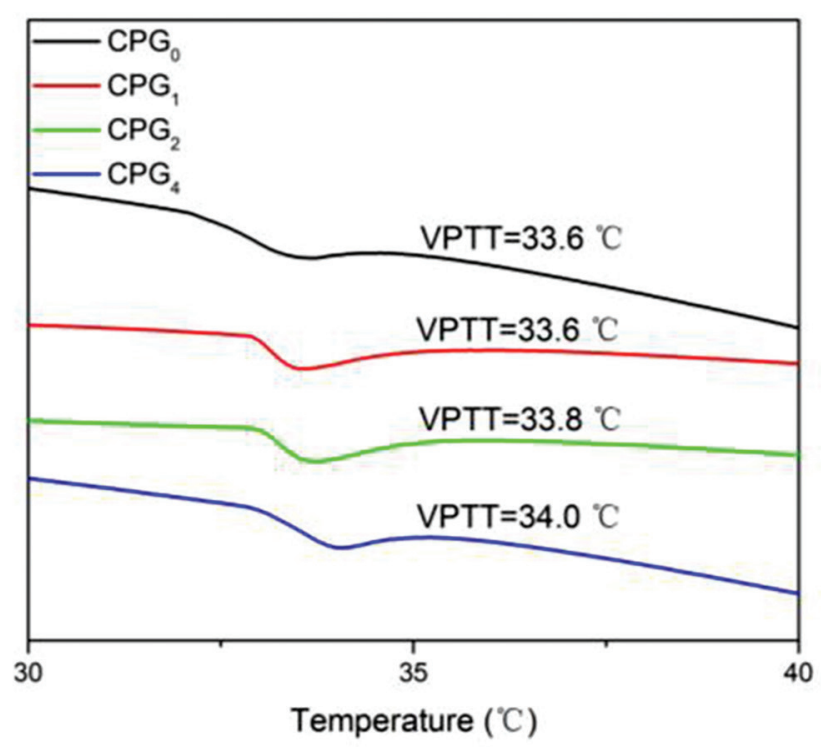

Figure 7: DSC curves of the hydrogel fibers.

Figure 8 shows the bending angle of hydrogel fibers within $60 \mathrm{~s}$ in deionized water at $50^{\circ} \mathrm{C}$. With increasing the GO content, both the bending angle and response rate of hydrogel fibers tend to decrease. The phenomenon is related to the mechanical properties and hydrophilicity of hydrogel fibers. As shown in Figure 4, the increase of GO content enhances the strength and modulus of the hydrogel fiber, making it more difficult to deform $(33,34)$. In addition, the hydrophilic groups on GO sheets and the compact polymer network structure lead to a decrease in the water lose rate of the hydrogel fiber.

\subsection{NIR light responsive bending behavior}

Figure $6 \mathrm{c}$ is the photographs of NIR light responsive bending of the $\mathrm{C} / \mathrm{CPG}_{2}$ fiber. The bilayer hydrogel fiber

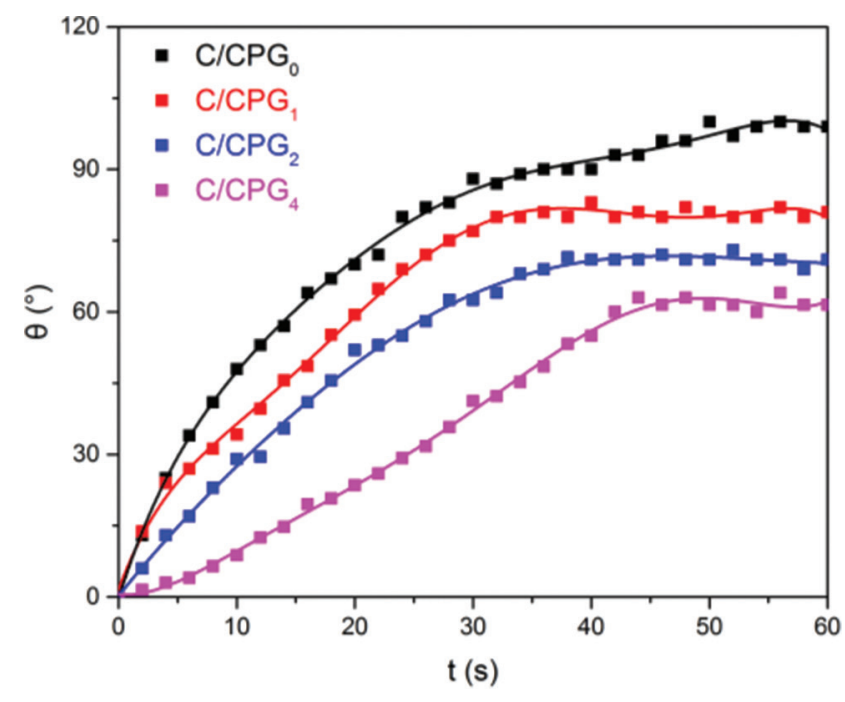

Figure 8: The bending angle of the hydrogel fibers in deionized water at $50^{\circ} \mathrm{C}$.

with length in $1.0 \mathrm{~cm}$ was fixed at one end and hung in the air. After applying NIR light, the bending angle of the bilayer hydrogel fiber was recorded. It can be seen that the hydrogel fiber also bent toward layer A, implying the shrinkage ability of layer $\mathrm{A}$ is higher than that of layer B. As well known, GO has a strong ability to absorb near infrared light and transform it into thermal energy. We measured the temperature of the hydrogels fiber after irradiated by NIR light. As shown in Figure 9, the irradiation of NIR light caused the increase in the temperature for bilayer hydrogel fibers with GO in layer A. For example, when the NIR light irradiation time was $5 \mathrm{~s}$, the temperature of $\mathrm{C} / \mathrm{CPG}_{4}$ fiber reached $38^{\circ} \mathrm{C}$ which was higher than its VPTT.

Such a phenomenon is caused by the efficient absorption and transformation of the NIR light energy to thermal energy by GO, which causes the temperature 
of layer A above its VPTT faster than the layer B as shown in Figure 9. Therefore, layer A shrank quickly and caused the bending of the bilayer hydrogel fiber. It can be concluded that GO in the hydrogel fibers can transfer energy and serve as a nanoheater to increase the temperature of the gel matrix uniformly and rapidly.

Figure 10 shows the bending angle of hydrogel fibers within $40 \mathrm{~s}$ after irradiated by NIR light. The bilayer hydrogel fibers show fast bending rate, the bending angle of $\mathrm{C} / \mathrm{CPG}_{4}$ fiber reaches $120^{\circ}$ within $30 \mathrm{~s}$. Moreover, the bending angle and response rate increase with increasing the GO content.

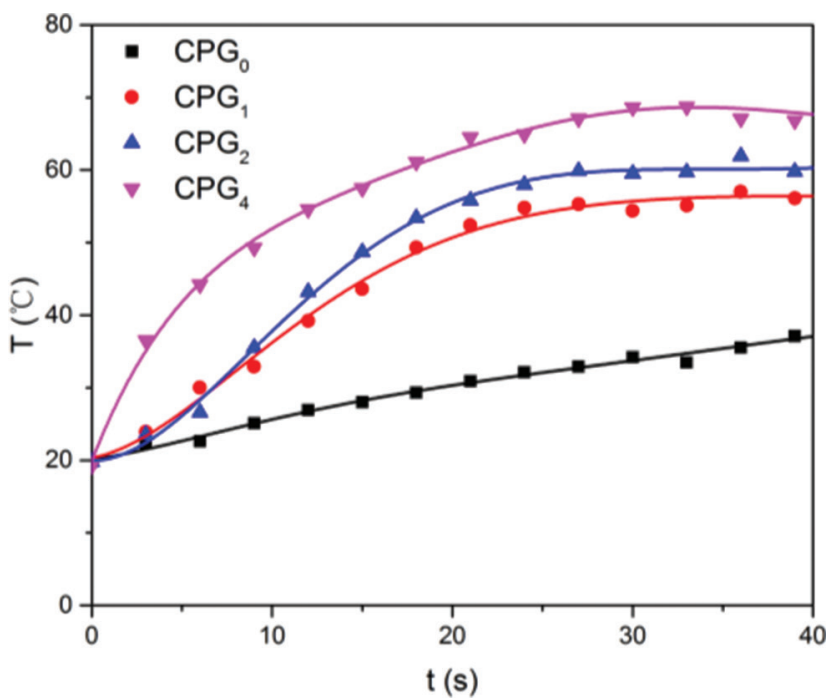

Figure 9: Temperature of the hydrogels fiber after irradiated by NIR light.

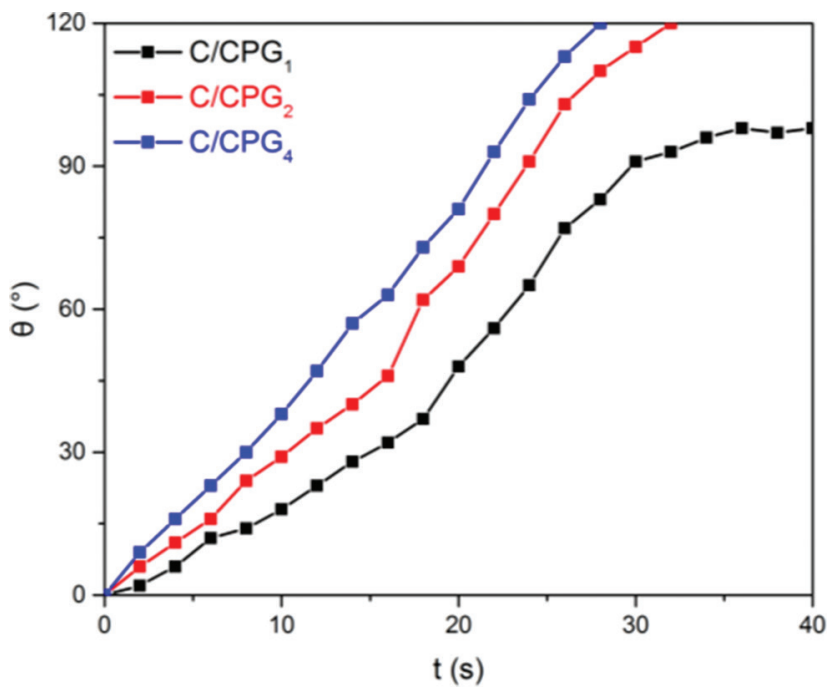

Figure 10: The bending angle of the hydrogel fibers after irradiated by NIR light.

\subsection{Influence of the spinning parameters on NIR light responsive bending behaviors}

In the progress of microfluidic spinning, the major factors for controlling the fiber diameter are the flow rates of the spinning solutions and the take-up velocity of winding device (35). As shown in Figure 11, under a fixed flow rate of outer fluid, the diameter of the hydrogel fiber increases almost linearly with increasing the flow rate of the inner fluids (A fluid and B fluid with the same flow rate). Similarly, the decrease in the flow rate can of outer fluid also increase the diameter of the hydrogel fiber, because the outer flow causes a shearing force on the inner flow during gel formation. In addition, Figure 11 show that under a fixed flow rate of inner and outer fluids, the increase in take-up velocity of winding device can also decrease the diameter of the hydrogel fiber. In summary, different diameters of hydrogel fibers can be obtained conveniently by changing the spinning parameters.

We also examined the effect of the diameter of the hydrogel fiber on the NIR light responsible and bending behavior. After being irradiated by NIR light, the hydrogel fibers with smaller diameters bend faster than those with larger diameters (Figure 12). For example, the bending angle of the hydrogel fiber with diameter of $320 \mu$ m reaches $120^{\circ}$ at $20 \mathrm{~s}$. The result is consistent with a literature report in which the shrinking/swelling rate of a stimuliresponsive hydrogel depends on its size because the $\mathrm{H}_{2} \mathrm{O}$ was expelled faster from the gel network (36).

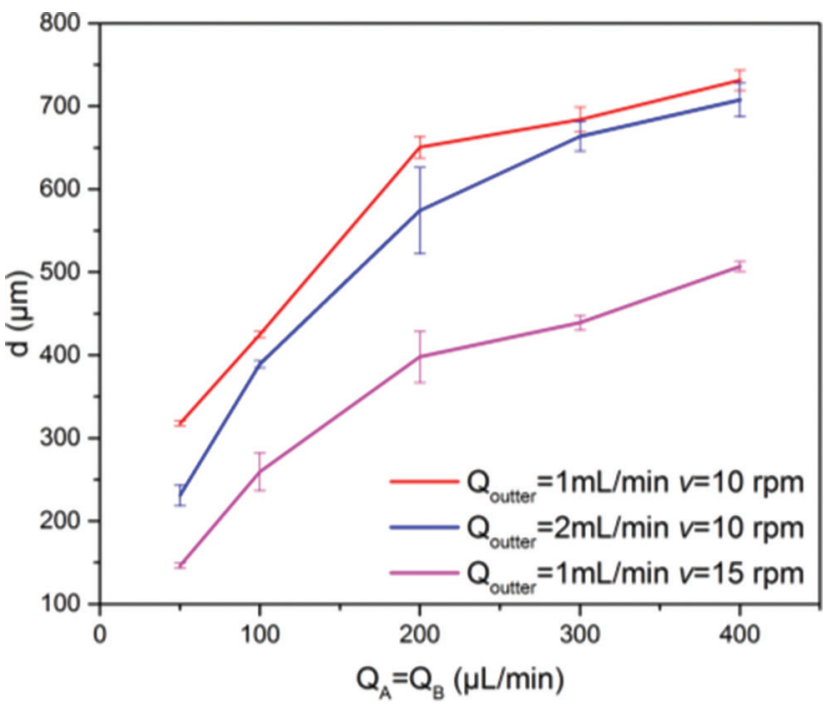

Figure 11: Influence of the flow rates of the A, B and outer fluids $\left(Q_{A}, Q_{B}\right.$ and $Q$ outter) and the take-up velocity of winding device (v) on the hydrogel fiber diameter. The A fluid and B fluid have the same flow rates. 


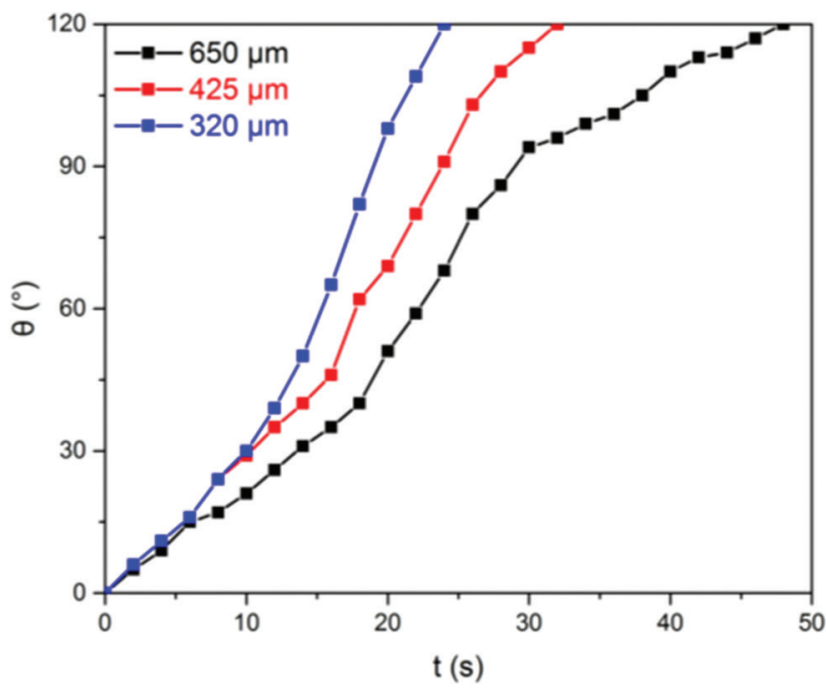

Figure 12: Effect of the diameter of $\mathrm{C} / \mathrm{CPG}_{4}$ fiber on the NIR light responsive bending behavior.

\section{Conclusions}

Here we report the preparation of bilayer hydrogel fibers based on a microfluidic spinning device. The bilayer hydrogel fiber can bend response to temperature and NIR light. In addition, the NIR light responsive property and mechanical property was increasing with the increasing of GO content. Besides, by changing the parameter of the spinning parameters, the diameter and NIR light responsive bending rate of hydrogel fibers can be controlled easily. Thus, the temperature/NIR light responsive hydrogel fiber can be applied in the NIR light driving actuators in the future.

Acknowledgements: This research used equipment located at the State Key Lab for Modification of Chemical Fibers and Polymer Materials, Donghua University. The authors are grateful to Li Peng (Donghua University) and Yan Liu (University of Twente) for their help in this study. The manuscript was written with contributions of all authors.

\section{References}

1. Liu Y., Zhang K., Ma J., Vancso G.J., Thermoresponsive semi-IPN hydrogel microfibers from continuous fluidic processing with high elasticity and fast actuation. ACS Appl Mat, 2017, 9(1), 901-908.

2. Zhang S., Bellinger A.M., Glettig D.L., Barman R., Lee Y.A., Zhu J., et al., A pH-responsive supramolecular polymer gel as an enteric elastomer for use in gastric devices. Nat Mater, 2015, 14(10), 1065-1071.
3. Peng L., Liu Y., Gong J., Zhang K., Ma J., Continuous fabrication of multi-stimuli responsive graphene oxide composite hydrogel fibres by microfluidics. RSC Advances, 2017, 7(31), 19243-19249.

4. Heo Y.J., Shibata H., Okitsu T., Kawanishi T., Takeuchi S., Long-term in vivo glucose monitoring using fluorescent hydrogel fibers. Proceedings of the National Academy of Sciences of the United States of America, 2011, 108(33), 13399-133403.

5. Ma Y.Y., Liu H.T., Ma J.H., Gong J.H., Glucose-responsive hydrogels based on phenylboronic acid. Materials Science Forum, 2018, 913, 714-721.

6. Sang W., Zhao L., Tang R., Wu Y., Zhu C., Liu J., Electrothermal actuator on graphene bilayer film. Macromol Mater Eng, 2017, 302(12), 1700239.

7. Zhang E.Z., Wang T., Hong W., Sun W.X., Liu X.X., Tong Z., Infrared-driving actuation based on bilayer graphene oxidepoly(N-isopropylacrylamide) nanocomposite hydrogels. J Mater Chem A, 2014, 2(37), 15633-15639.

8. Nakajima S., Kawano R., Onoe H., Stimuli-responsive hydrogel microfibers with controlled anisotropic shrinkage and crosssectional geometries. Soft Matter, 2017, 13(20), 3710-3719.

9. Cheng Y., Zheng F.Y., Lu J., Shang L.R., Xie Z.Y., Zhao Y.J., et al., Bioinspired multicompartmental microfibers from microfluidics. Adv Mater, 2014, 26(30), 5184-5190.

10. Gernhardt M., Peng L., Burgard M., Jiang S., Förster B., Schmalz H., et al., Tailoring the morphology of responsive bioinspired bicomponent fibers. Macromol Mater Eng, 2018, 303(1), 1700248.

11. Jiang S., Liu F., Lerch A., Ionov L., Agarwal S., Unusual and superfast temperature-triggered actuators. Adv Mater, 2015, 27(33), 4865-4870.

12. Liu L., Bakhshi H., Jiang S., Schmalz H., Agarwal S., Composite polymeric membranes with directionally embedded fibers for controlled dual actuation. Macromol Rapid Comm, 2018, 39(10), 1800082.

13. Asoh T.A., Matsusaki M., Kaneko T., Akashi M., Fabrication of temperature-responsive bending hydrogels with a nanostructured gradient. Adv Mater, 2008, 20(11), 2080-2083.

14. Peng Q., Shao H., Hu X., Zhang Y., The development of fibers that mimic the core-sheath and spindle-knot morphology of artificial silk using microfluidic devices. Macromol Mater Eng, 2017, 302(10), 1700102.

15. Zhang X., Pint C.L., Lee M.H., Schubert B.E., Jamshidi A., Takei K., et al., Optically and thermally-responsive programmable materials based on carbon nanotube-hydrogel polymer composites. Nano Lett, 2011, 11(8), 3239-3244.

16. Wang E., Desai M.S., Lee S.W., Light-controlled grapheneelastin composite hydrogel actuators. Nano Lett, 2013, 13(6), 2826-2830.

17. Shi K., Liu Z., Wei Y.Y., Wang W., Ju X.J., Xie R., et al., Near infrared light-responsive poly(N-isopropylacrylamide)/graphene oxide nanocomposite hydrogels with ultrahigh tensibility. ACS Appl Mat, 2015, 7(49), 27289-27298.

18. Wen L., Jiasi W., Jinsong R., Qu X., 3D graphene oxide-polymer hydrogel: near infrared light triggered active scaffold for reversible cell capture and on demand release. Adv Mater, 2013, 25(46), 6737-6743.

19. Huang X., Neretina S., Sayed M.A.E., Gold nanorods: from synthesis and properties to biological and biomedical applications. Adv Mater, 2009, 21(48), 4880-4910. 
20. Yang X., Liu X., Liu Z., Pu F., Ren J., Near infrared light triggered, targeted drug delivery to cancer cells by aptamer gated nanovehicles. Adv Mater, 2012, 24(21), 2890-2895.

21. Zhu C., Lu Y., Chen J., Yu S., Photothermal poly(N-isopropylacrylamide) $/ \mathrm{Fe}_{3} \mathrm{O}_{4}$ nanocomposite hydrogel as a movable position heating source under remote control. Small, 2014, 10(14), 2796-2800.

22. Fusco S., Sakar M.S., Kennedy S., Peters C., Bottani R., Starsich F., et al., An integrated microrobotic platform for on demand, targeted therapeutic interventions. Adv Mater, 2014, 26(6), 952-957.

23. Fares M.M., Othman A.A., Lower critical solution temperature determination of smart, thermosensitive $\mathrm{N}$-isopropylacrylamide-alt-2-hydroxyethyl methacrylate copolymers: kinetics and physical properties. J Appl Polym Sci, 2008, 110(5), 2815-2825.

24. Li Y., Zhang X., Cheng H., Zhu J., Cheng S., Zhuo R., Self-assembled, thermosensitive PCL-g-P(NIPAAm-co-HEMA) micelles for drug delivery. Macromol Rapid Comm, 2006, 27(22), 1913-1919.

25. Zhang B., Sun S., Wu P., Synthesis and unusual volume phase transition behavior of poly( $\mathrm{N}$-isopropylacrylamide)-poly(2hydroxyethyl methacrylate) interpenetrating polymer network microgel. Soft Matter, 2013, 9(5), 1678-1684.

26. Cheng H., Shen L., Wu C., LLS and FTIR studies on the hysteresis in association and dissociation of poly( $\mathrm{N}$-isopropylacrylamide) chains in water. Macromolecules, 2006, 39(6), 2325-2329.

27. Fetters L.J., Hadjichristidis N., Lindner J.S., Mays J.W., Molecular weight dependence of hydrodynamic and thermodynamic properties for well defined linear polymers in solution. J Phys Chem Ref Data, 1994, 23(4), 619-640.
28 Braccini I., Pérez S., Molecular basis of $\mathrm{Ca}^{2+}$-induced gelation in alginates and pectins: the egg-box model revisited. Biomacromolecules, 2001, 2(4), 1089-1096.

29. Ma X., Li Y., Wang W., Ji Q., Xia Y., Temperature-sensitive poly(N-isopropylacrylamide)/graphene oxide nanocomposite hydrogels by in situ polymerization with improved swelling capability and mechanical behavior. Eur Polym J, 2013, 49(2), 389-396.

30. Shen J., Yan B., Li T., Long Y., Li N., Ye M., Study on graphene oxide based polyacrylamide composite hydrogels. Compos Part A-Appl S, 2012, 43(9), 1476-1481.

31. Zhang H., Zhai D., He Y., Graphene oxide/polyacrylamide/ carboxymethyl cellulose sodium nanocomposite hydrogel with enhanced mechanical strength: preparation, characterization and the swelling behavior. RSC Adv, 2014, 4(84), 44600-44609.

32. Ping C.H., Ping W., Hong Y.S., Highly elastic and superstretchable graphene oxide/polyacrylamide hydrogels. Small, 2014, 10(3), 448-453.

33. Guo W., Li M., Zhou J., Modeling programmable deformation of self-folding all-polymer structures with temperature-sensitive hydrogels, Smart Mater Struct, 2013, 22(11), 115028

34 Yarin A.L., Agarwal S., Buckling and unraveling poly(N-isopropyl acrylamide)-thermoplastic polyurethane bilayers. Polymer, 2016, 97, 604-613.

35. Jeong W., Kim J., Kim S., Lee S., Mensing G., Beebe D.J., Hydrodynamic microfabrication via "on the fly" photopolymerization of microscale fibers and tubes. Lab Chip, 2004, 4(6), 576-580.

36. Tanaka T., Fillmore D.J., Kinetics of swelling of gels. J Chem Phys, 1979, 70(3), 1214-1218. 